\title{
Model and system learners, optimal process constructors and kinetic theory-based goal-oriented design: A new paradigm in materials and processes informatics
}

Emmanuelle Abisset-Chavanne, Jean Louis Duval, Elias Cueto, and Francisco Chinesta

Citation: AIP Conference Proceedings 1960, 090004 (2018); doi: 10.1063/1.5034930

View online: https://doi.org/10.1063/1.5034930

View Table of Contents: http://aip.scitation.org/toc/apc/1960/1

Published by the American Institute of Physics

\section{Articles you may be interested in}

A cyber physical system approach for composite part: From smart manufacturing to predictive maintenance AIP Conference Proceedings 1960, 020025 (2018); 10.1063/1.5034826

Data-driven in computational plasticity

AIP Conference Proceedings 1960, 090006 (2018); 10.1063/1.5034932

Consistent data-driven computational mechanics

AIP Conference Proceedings 1960, 090005 (2018); 10.1063/1.5034931

Simulation of the microwave heating of a thin multilayered composite material: A parameter analysis AIP Conference Proceedings 1960, 020035 (2018); 10.1063/1.5034836

Prediction of composites behavior undergoing an ATP process through data-mining AIP Conference Proceedings 1960, 020016 (2018); 10.1063/1.5034817

Microwave heating for thermoplastic composites - Could the technology be used for welding applications? AIP Conference Proceedings 1960, 020003 (2018); 10.1063/1.5034804 


\title{
Model and system learners, optimal process constructors and kinetic theory-based goal-oriented design: a new paradigm in materials and processes informatics
}

\author{
Emmanuelle Abisset-Chavanne ${ }^{1, \mathrm{~b})}$, Jean Louis Duval ${ }^{2, \mathrm{c})}$, Elias Cueto $^{3, \mathrm{~d})}$ and \\ Francisco Chinesta ${ }^{4, a), e)}$
}

\author{
${ }^{1}$ ESI GROUP Chair E ICI Institute, Centrale Nantes, 1 Rue de la Noe, F-44300 Nantes, France \\ ${ }^{2}$ ESI GROUP, 99 rue des Solets, F-94513 Rungis, France \\ ${ }^{3}$ Aragón Institute of Engineering Research, Universidad de Zaragoza, Mara de Luna, s.n., F-50018 Zaragoza, Spain \\ ${ }^{4}$ ESI GROUP Chair E PIMM, ENSAM ParisTech, 151 Boulevard de l'Hôpital, F-75013 Paris, France
}

\author{
${ }^{a)}$ Corresponding author: Francisco.Chinesta@ec-nantes.fr

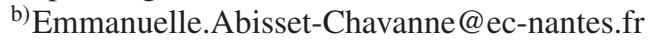 \\ c)Jean-Louis.Duval@esi-group.com \\ d)ecueto@unizar.es \\ e)Francisco.Chinesta@ec-nantes.fr
}

\begin{abstract}
Traditionally, Simulation-Based Engineering Sciences (SBES) has relied on the use of static data inputs (model parameters, initial or boundary conditions, ... obtained from adequate experiments) to perform simulations. A new paradigm in the field of Applied Sciences and Engineering has emerged in the last decade. Dynamic Data-Driven Application Systems [9, 10, 11, 12, 22] allow the linkage of simulation tools with measurement devices for real-time control of simulations and applications, entailing the ability to dynamically incorporate additional data into an executing application, and in reverse, the ability of an application to dynamically steer the measurement process. It is in that context that traditional "digital-twins" are giving raise to a new generation of goal-oriented data-driven application systems, also known as "hybrid-twins", embracing models based on physics and models exclusively based on data adequately collected and assimilated for filling the gap between usual model predictions and measurements. Within this framework new methodologies based on model learners, machine learning and kinetic goal-oriented design are defining a new paradigm in materials, processes and systems engineering.
\end{abstract}

\section{INTRODUCTION}

Virtual twins emulating a physical system from the accurate solution of the mathematical model expected describing it, were major protagonists in most of scientific fields and engineering domains, making possible the virtual evaluation of systems responses. However, usually virtual twins are static, that is, they are used in the design of complex systems and their components, but they are not expected to accommodate or assimilate data so as to define dynamic data-driven application systems. The reason is that the characteristic time of standard simulation strategies is not compatible with the real-time constraints compulsory for control purposes. Thus, real-time control was ensured by techniques based on the use of simplified representations of the system. One could think that all these issues could be circumvented with the mere use of more powerful computers, however such a route compromises its integration in deployed systems.

New numerical technologies based on Model Order Reduction - MOR - techniques opened new possibilities for more efficient simulations. An appealing route consists in calculating offline the parametric solution of a given model containing the solution of all possible scenarios, to be then particularized online using light computational facilities, as deployed devices, for performing efficient simulation, optimization, inverse analysis, uncertainty propagation and simulation-based control, all them under real-time constraints. This was the solution retained in many applications where the so-called PGD - Proper Generalized Decomposition - was involved $[1,2,4,5,6,7,8]$. 
These new simulation techniques are at the basis of the so-called digital twins that allowed assimilating data collected from sensors with as main aim the one of identifying parameters involved in the model as well as their time evolution in real time, anticipating actions from their predictive capabilities.

Despite an initial euphoric and jubilant period in which high-fidelity models were examined at all their scales in almost real-time by using reasonable computing platforms and efforts, when these computing facilities were integrated into data-driven applications systems unexpected difficulties appeared immediately. Namely, in practice significant deviations between the predicted and observed responses were noticed, limiting their use in many applications.

The origin of the just referred deviations between predictions and measurements is due to: (i) inaccuracies in the employed models that sometimes continue to be crude descriptions of the real systems; (ii) to the fact that in many cases models evolve in time in an "a priori" almost unpredictable manner; and (iii) inaccuracies in the determination of the model parameters or in their time-evolution, that can present space and time stochastic fluctuations. A certain part of the deviation (its unbiased component in a statistical sense) can be viewed as a noise. However, the remaining biased part proves the existence of a hidden model that operates but escapes to our understanding. In order to address this inevitable "ignorance" one possibility lies in constructing "on-the-fly" a data-driven model able to fill the gap between prediction and measurement. Only when this gap disappears, system control can be efficiently attained. This is the rationale defining the concept of Hybrid Twin.

\section{FROM DIGITAL TWINS TO HYBRID TWINS}

\section{The Digital Twin}

A given physical system is characterized by a number of continuous or discrete variables. In general, to manipulate these variables in a computer, continuous variables are discretized, i.e. more than looking for those variable at any point, it is assumed that variables at any point can be expressed from the ones existing in some particular locations (the nodes using the finite element terminology) using adequate interpolations.

In what follows the discrete form of the variables defining the system state at time $t$ is denoted by $\mathbf{X}(t) \in \mathbb{R}^{D}$. As just indicated, they could contain depending on the considered physics, nodal temperatures, velocities, displacements, stresses, etc.

The system evolution is described by its state $\mathbf{X}(t)$ evolving from its initial state at the initial time $t=t_{0}=0$, denoted by $\mathbf{X}_{0}$. Numerical models based on well established physics allow making this prediction of the system state at time $t$ from the knowledge of it at the initial time $t_{0}$, by integrating its rate of change (coming from the physical laws) given by $\dot{\mathbf{X}}(\tau)$ in $\tau \in(0, t]$.

This contribution, when emphasizing its parametric form will be expressed by $\dot{\mathbf{X}}(t ; \boldsymbol{\mu})=\mathbf{A}(\mathbf{X}, t ; \boldsymbol{\mu})$, where $\boldsymbol{\mu}$ represent the set of parameters that the model involves and that should be identified offline or online. In the previous expression the semicolon $(\cdot ; \cdot)$ makes a distinction between the coordinates before the semicolon - in this case the time - and the model parameters after it - here $\boldsymbol{\mu}$-.

When identification is performed online, model parameters $\boldsymbol{\mu}$ are calculated by enforcing that the associated model prediction fits as much as possible experimental measurements, at least at the measurements points. In the context of model calibration, data was needed for identifying, offline or online, the parameters involved in the model.

Thus, when models were assumed representing accurately the subjacent physics involved in the system, predictions were easily performed by computing the solution by integrating $\mathbf{A}(\mathbf{X}, t ; \boldsymbol{\mu})$. Finite elements, finite differences, finite volumes, spectral methods, meshless (or meshfree) techniques, ... are some of the techniques well experienced and widely used for solving the complex mathematical models by discretizing them.

In the context of process or system control, external actions are applied for driving the model solution towards the given target. Thus, the state rate of change (when ignoring noise) is composed by two contributions:

$$
\dot{\mathbf{X}}(t ; \boldsymbol{\mu})=\mathbf{A}(\mathbf{X}, t ; \boldsymbol{\mu})+\mathbf{C}(t),
$$

whose online integration compromises real-time feedback.

This severe limitation was alleviated by using model order reduction techniques as discussed in the previous section. In particular, the Proper Generaliazed Decomposition - PGD - precomputed (offline) the parametric solution making possible to accommodate real-time constraints. 


\section{The Hybrid Twin}

When models roughly represent the associated physics, a non negligible deviation between their predictions and the real evolution acquired from collected data is expected. This deviation is expected biased because it represents the modeler ignorance on the subjacent physics. The unbiased deviation contribution is associated to modeling or measurement noise and is easily addressed by using adequate filters, however, biased deviations express hidden physics and required a particular treatment, that is, their online modeling by assimilating collected data.

Indeed, the deviation (gap between the model prediction $\mathbf{X}(t)$ and measurements $\mathbf{X}^{\exp }(t)$ ) for the optimal choice of the model parameters $\boldsymbol{\mu}$, i.e. $\mathbf{X}(t)-\mathbf{X}^{\exp }(t)$, and more concretely its time derivative, should be used for the online construction (under the severe real-time constraint) of the so-called data-based correction model (also referred as deviation model) denoted by $\mathbf{B}(\mathbf{X}, t)$. Even if in what follows the presence of unbiased noise is ignored, its inclusion is straightforward.

The recent exponential growing of machine learning techniques (data-mining, deep-learning, manifold learning, ... for citing few) makes possible to construct on-the-fly such a data-based deviation model that allows writing the fundamental equation of an hybrid twin:

$$
\dot{\mathbf{X}}(t ; \boldsymbol{\mu})=\mathbf{A}(\mathbf{X}, t ; \boldsymbol{\mu})+\mathbf{B}(\mathbf{X}, t)+\mathbf{C}(t)+\mathbf{R}(t),
$$

expressing that the rate of change of the system state at time $t$ contains four main contributions:

- (i) the pre-assumed physical contribution whose state rate of change related to the model parameters $\boldsymbol{\mu}$ reads $\mathbf{A}(\mathbf{X}, t ; \boldsymbol{\mu})$;

- (ii) a data-based model $\mathbf{B}(\mathbf{X}, t)$ modeling the noticed gaps between prediction and measurement;

- (iii) external actions $\mathbf{C}(t)$ introduced into the system dynamics in order to drive the model solution towards the desired target;

- (iv) the unbiased noise $\mathbf{R}(t)$ that was traditionally addressed using appropriate filters [15].

Remark. In some circumstances the physical model is almost unattainable and then the physical contribution $\mathbf{A}$ is absent. Thus, the only contribution concerns the data-based model that is constructed from the scratch by using any of the available techniques of machine learning. Models based on regression, model learners or neural-networks (deep learning) constitute some of the most usual approaches to model this kind of systems.

In the previous expression (2), the data-based contribution $\mathbf{B}(\mathbf{X}, t)$ makes the difference between digital twins (whose main characteristic is assimilating measurements provided by sensors), and hybrid twins, the last representing the real system all along its life, adapting it to any noticed deviation and keeping all its predictive capacity, necessary for control purposes and real-time decision making. It is usually argued that data has as main difficulty the extrapolation, whereas model have such facility. However hybrid twins defined models from data, and consequently they recover a capacity which had been unjustifiably rejected.

\section{ADVANCED METHODS}

\section{Data-Based Model: Model Learners}

Different possibilities exist for constructing the data-driven correction contribution to the dynamical system $\mathbf{B}(\mathbf{X})$. Sparse identification $[3,18]$ considers that this unknown contribution can be expressed by using a set of almost arbitrary functions, and different combinations of them. It is expected that the solution does not require all of them, and consequently $L^{1}$-optimization could be considered because the solution in that basis is expected being sparse. Many other possibilities exist: regression-based models (many times based on the use of decision trees or random forest), neural network-based deep-learning, ...

In the case of a dynamical system we assume the rate of change known at $S$ different system states, that is $\left\{\dot{\mathbf{X}}_{1}, \ldots, \dot{\mathbf{X}}_{\mathrm{S}}\right\}$ at states $\left\{\mathbf{X}_{1}, \ldots, \mathbf{X}_{\mathrm{S}}\right\}$, all them defined in $\mathbb{R}^{D}$. Now, the model linking inputs (state) with outputs (state rate of change) has the discrete form consisting of matrix $\mathbf{M}(\mathbf{X})$, ensuring $\dot{\mathbf{X}}=\mathbf{M}(\mathbf{X}) \mathbf{X}$, from which matrix $\mathbf{M}(\mathbf{X})$ can be identified in a moving least squares sense.

Another possibility consists of approximating locally and linearly the manifold or its reduced counterpart. In that case we assume that we can collect again numerous state snapshots $\left\{\mathbf{X}_{1}, \ldots, \mathbf{X}_{\mathrm{S}}\right\}$, from which obtaining the slow 
manifold containing the different images, $\left\{\boldsymbol{\xi}_{1}, \ldots, \boldsymbol{\xi}_{\mathrm{s}}\right\}[13,16,17,19,20]$. Now, to each point $\mathbf{X}_{i}$, we associate a label consisting of its rate of change $\dot{\mathbf{X}}_{i}$, and this label is transferred to its image $\boldsymbol{\xi}_{i}$. In order to express the rates $\dot{\mathbf{X}}_{i}$ as functions of the reduced coordinates $\boldsymbol{\xi}$, we consider

$$
\dot{\mathbf{X}}=\frac{\partial \mathbf{X}}{\partial \boldsymbol{\xi}} \dot{\boldsymbol{\xi}} \rightarrow \dot{\boldsymbol{\xi}}=\left(\frac{\partial \mathbf{X}}{\partial \boldsymbol{\xi}}\right)^{-1} \dot{\mathbf{X}}
$$

that allows replacing the known rates $\dot{\mathbf{X}}_{i}$ by theirs associated reduced rates $\dot{\boldsymbol{\xi}}_{i}$.

Thus, we obtain finally $\mathrm{S}$ reduced couples $\left(\dot{\boldsymbol{\xi}}_{i}, \boldsymbol{\xi}_{i}\right), i=1, \ldots, \mathrm{S}, \boldsymbol{\xi}_{i} \in \mathbb{R}^{d}, d \ll D$, one can learn the local reduced model $\mathbf{m}(\boldsymbol{\xi})$, such that $\dot{\xi}=\mathbf{m}(\boldsymbol{\xi}) \boldsymbol{\xi}$, again in moving least square sense.

\section{PGD-Based Parametric Solution}

When considering the dynamical system $\dot{\mathbf{X}}=\mathbf{A}(\mathbf{X}, t, \boldsymbol{\mu})+\mathbf{C}(\mathbf{X})+\mathbf{R}$, where here $\mathbf{A}$ is assumed incorporating the data-based learned model, involving parameters $\mu_{i}, i=1, \cdots, \mathbf{Q}$, and assuming that the control is defined from a dictionary of actions implying weights $\gamma_{k}, k=1, \ldots, \mathrm{C}$; PGD makes possible computing the parametric updated state $\mathbf{X}\left(t+\Delta t, \mu_{1}, \ldots, \mu_{\mathrm{Q}}, \gamma_{1}, \ldots, \gamma_{\mathrm{C}}, \mathbf{X}(t)\right)$. However, as the initial state $\mathbf{X}(t)$ involved into the updating at time $t+\Delta t$ remains in practice too rich, it is also expressed in an appropriate dictionary (e.g. POD modes) involving reconstruction weights $\delta_{l}, l=1, \ldots, \mathrm{D}$, that allows rewritten the previous parametric state updating as $\mathbf{X}\left(t+\Delta t, \mu_{1}, \ldots, \mu_{Q}, \gamma_{1}, \ldots, \gamma_{\mathrm{C}}, \delta_{1}, \ldots, \delta_{\mathrm{D}}\right)$, that could be successfully computed despite its high-dimensionality by using the separate representation constructor [5]. A similar approach was consider in [14].

\section{Kinetic Theory Formalism}

It is well known that stochastic equations can be inserted into a kinetic theory formalism, leading to the so-called Fokker-Planck equation. For that purpose we introduce the probability distribution function - pdf $-\Psi(\mathbf{x}, t)$ giving the probability of having the state $\mathbf{x} \in \mathbb{R}^{D}$ at time $t$, and its associated Fokker-Planck equation that reads

$$
\frac{\partial \Psi}{\partial t}+\frac{\partial}{\partial \mathbf{x}}(\dot{\mathbf{x}} \Psi)=0 .
$$

Then, by rewritting the dynamic equation as $\dot{\mathbf{X}}=\mathbf{M}(\mathbf{X}) \mathbf{X}+\mathbf{R}=\mathbf{K}(\mathbf{X})+\mathbf{R}$, with $\mathbf{R}=\mathbf{G W}$, with $\mathbf{W}$ a Ddimensional Wiener process (remember that $\mathbf{X} \in \mathbb{R}^{D}$ ), from which the Fokker-Planck equation can be rewritten as

$$
\frac{\partial \Psi}{\partial t}+\frac{\partial}{\partial \mathbf{x}}(\mathbf{K}(\mathbf{x}, t) \Psi)=\frac{\partial}{\partial \mathbf{x}}\left(\mathbf{G G}^{T} \frac{\partial \Psi}{\partial \mathbf{x}}\right) .
$$

Expressing the Hybrid-Twin from a kinetic theory formalism has numerous advantages:

1. Independently of the linear or nonlinear character of the dynamic equation, the Fokker-Planck equation is linear.

2. In the solution of equation (5) coexists all possible trajectories of the stochastic equation.

3. The Fokker-Planck formalism allows to perform data-assimilation within a Bayesian framework [21].

4. Even if the steady solution of the dynamical system has not really sense, in some circumstances it exists a steady state pdf.

5. Calculating sensibilities of a dynamical system is a tricky issue because the Brownian fluctuations. However, it become easier when proceeding from its kinetic theory counterpart.

6. Approximating the pdf requires when proceeding in the stochastic framework the calculation of (too) many trajectories of the stochastic process with the associated computational cost, even if the calculation of its moments requires much less trajectories.

The main difficulty in considering the solution of the Fokker-Planck equation is associated to its highdimensionality. 


\section{Nonlinear Dimensionality Reduction}

To circumvent the curse of dimensionality one is tempted to consider nonlinear dimensionality reduction previously discussed when addressing model learning, with the aim of reducing the dimension of the state space.

By defining the Jacobian

$$
\mathbf{J}(\xi)=\frac{\partial \mathbf{X}(\xi)}{\partial \xi} .
$$

the reduced dynamical system can be rewritten as

$$
\dot{\xi}=\frac{\partial \xi}{\partial X} \dot{\mathbf{X}}=\mathbf{J}^{-1}(\boldsymbol{\xi}) \dot{\mathbf{X}}=\mathbf{J}^{-1}(\boldsymbol{\xi}) \mathbf{K}(\mathbf{X}(\boldsymbol{\xi})) .
$$

that allows writing the reduced Fokker-Planck equation involving $\Psi(\xi, t)$

$$
\frac{\partial \Psi}{\partial t}+\frac{\partial}{\partial \boldsymbol{\xi}}\left(\mathbf{J}^{-1}(\boldsymbol{\xi}) \mathbf{K}(\mathbf{X}(\boldsymbol{\xi})) \Psi\right)=\frac{\partial}{\partial \boldsymbol{\xi}}\left(\mathbf{g g}^{T} \frac{\partial \Psi}{\partial \xi}\right),
$$

where $\mathbf{g}=\mathbf{J}^{-1} \mathbf{G}$.

\section{Steady-State Solutions and Goal Oriented Dynamical Systems}

When considering the Fokker-Planck equation

$$
\frac{\partial \Psi}{\partial t}+\frac{\partial}{\partial \mathbf{x}}(\mathbf{K}(\mathbf{x}, t) \Psi)=\frac{\partial}{\partial \mathbf{x}}\left(\mathbf{G G}^{T} \frac{\partial \Psi}{\partial \mathbf{x}}\right)
$$

different situations can be found:

- If $\mathbf{K}(\mathbf{x})$ is time-independent, the steady state results from

$$
\frac{\partial}{\partial \mathbf{x}}(\mathbf{K}(\mathbf{x}) \Psi)=\frac{\partial}{\partial \mathbf{x}}\left(\mathbf{G G}^{T} \frac{\partial \Psi}{\partial \mathbf{x}}\right),
$$

and two possibilities exist: (i) if $\mathbf{K}(\mathbf{x})$ is (assumed) known, the steady state results from the solution of Eq. (10); and (ii) if the pdf $\Psi$ is assumed known (target distribution), Eq. (10) could be used to determine the optimal dynamical system $\mathbf{K}(\mathbf{x})$, that should be then associated to a physical system.

- If $\mathbf{K}(\mathbf{x}, t)$ is time-dependent, the existence of a steady state is seriously compromised and does not exist in the general case. Thus the associated Fokker-Planck equation consists in the most general expression,

$$
\frac{\partial \Psi}{\partial t}+\frac{\partial}{\partial \mathbf{x}}(\mathbf{K}(\mathbf{x}, t) \Psi)=\frac{\partial}{\partial \mathbf{x}}\left(\mathbf{G G}^{T} \frac{\partial \Psi}{\partial \mathbf{x}}\right) .
$$

In a forced regime, a steady-state in the frequency domain could exist and, as in such a long-time solution the initial condition vanishes, the fact of moving to the Fourier space is fully justified. In this case, Eq. (11) becomes

$$
i \omega \hat{\Psi}+\frac{\partial}{\partial \mathbf{x}}(\widehat{\mathbf{K \Psi}})=\frac{\partial}{\partial \mathbf{x}}\left(\mathbf{G G}^{T} \frac{\partial \hat{\Psi}}{\partial \mathbf{x}}\right),
$$

with $\hat{\Psi}(\mathbf{x}, \omega)=\mathcal{F}(\Psi(\mathbf{x}, t))$, being the Fourier transform of the pdf. Thus, a steady-state solution could exist in the frequency domain, implying a periodic solution in the time domain (containing eventually many frequencies because the Fournier transform $\widehat{\mathbf{K \Psi}}$ mixes the frequencies involved in the dynamic term).

\section{Conclusions}

The present work proposes a generic framework for a new paradigm for advanced dynamic data-driven application systems that embraces physically based models with models based exclusively on data. Different new numerical tools have been proposed for addressing the main issues related to such a procedure. Finally, when inserting the general framework within a kinetic theory format new unexpected possibilities for advanced goal-oriented design appear, that could be inserted into a kinetic evolutionary framework as proposed in [23] (that constitutes a work in progress). 


\section{REFERENCES}

[1] A. Ammar, B. Mokdad, F. Chinesta, R. Keunings, A new family of solvers for some classes of multidimensional partial differential equations encountered in kinetic theory modeling of complex fluids. J. Non-Newton. Fluid Mech. 139, 153-176 (2006).

[2] A. Ammar, B. Mokdad, F. Chinesta, R. Keunings. A new family of solvers for some classes of multidimensional partial differential equations encountered in kinetic theory modeling of complex fluids. Part II: Transient simulation using space-time separated representation. J. Non-Newton. Fluid Mech. 144, 98-121 (2007).

[3] S. Brunton, J. Proctor, N. Kut. Discovering governing equations from data by sparse identification of nonlinear dynamical systems. PNAS, 113/15, 3932-3937 (2016).

[4] F. Chinesta, P. Ladeveze, E. Cueto. A short review in model order reduction based on proper generalized decomposition. Arch. Comput. Methods Eng. 18, 395-404 (2011).

[5] F. Chinesta, A. Leygue, F. Bordeu, J.V. Aguado, E. Cueto, D. Gonzalez, I. Alfaro, A. Ammar, A. Huerta. PGDbased computational vademecum for efficient design, optimization and control. Arch. Comput. Methods Eng. 20, 31-59 (2013).

[6] F. Chinesta, R. Keunings, A. Leygue. The Proper Generalized Decomposition for Advanced Numerical Simulations. A primer, (Springerbriefs, Springer, 2014).

[7] F. Chinesta, P. Ladeveze (Edts). Separated Representations and PGD Based Model Reduction: Fundamentals and Applications, (CISM-Springer, 2014).

[8] F. Chinesta, A. Huerta, G. Rozza, K. Willcox. 'Model Order Reduction" in Encyclopedia of Computational Mechanics, (Second Edition, Erwin Stein, René de Borst \& Tom Hughes Edt., John Wiley \& Sons Ltd., 2015).

[9] F. Darema. "Engineering/scientific and commercial applications: differences, similarities, and future evolution" in Proceedings of the Second Hellenic European Conference on Mathematics and Informatics, 1994, pp. 367-374.

[10] "DDDAS Workshop 2006 Final Report" in Arlington, VA, USA, Technical report, National Science Foundation, 2006.

[11] "DDDAS Workshop 2000 Final Report" in Arlington, VA, USA, Technical report, National Science Foundation, 2000.

[12] Ch. Ghnatios, F. Masson, A. Huerta, E. Cueto, A. Leygue, F. Chinesta. Proper generalized decomposition based dynamic data-driven control of thermal processes. Comput. Methods Appl. Mech. Eng. 213, 29-34 (2012).

[13] D. Gonzalez, J.V. Aguado, E. Cueto, E. Abisset, F. Chinesta. kPCA-based parametric solutions within the PGD framework. Archives of Computational Methods in Engineering 25/1, 6986, 2018.

[14] D. Gonzalez, E. Cueto, F. Chinesta. Real-time direct integration of reduced solid dynamics equations. International Journal for Numerical Methods in Engineering 99/9, 633653 (2014).

[15] D. Gonzalez, A. Badias, I. Alfaro, F. Chinesta, E. Cueto. Model order reduction for real-time data assimilation through Extended Kalman Filters. Computer Methods in Applied Mechanics and Engineering 326/1 679-693 (2017).

[16] R. Ibanez, E. Abisset-Chavanne, J.V. Aguago, D. Gonzalez, E. Cueto, F. Chinesta. A manifold learning approach to data-driven computational elasticity and inelasticity, Archives of Computational Methods in Engineering 25/1, 4757 (2018).

[17] R. Ibanez, D. Borzacchiello, J.V. Aguado, E. Abisset-Chavanne, E. Cueto, P. Ladeveze, F. Chinesta. Datadriven non-linear elasticity. Constitutive manifold construction and problem discretization. Computational Mechanics 60/5, 813826 (2017).

[18] J.N. Kutz. Data-driven modeling E scientific computation. Methods for complex systems $\mathcal{E}$ big-data (Oxford University Press, 2013).

[19] J.A. Lee, M. Verleysen. Nonlinear dimensionality reduction (Springer, Berlin, 2007).

[20] E. Lopez, D. Gonzalez, J.V. Aguado, E. Abisset, E. Cueto, C. Binetruy, F. Chinesta. A manifold learning approach for integrated computational materials engineering. Archives of Computational Methods in Engineering 25/1, 5968, 2018.

[21] R.N. Miller, E.F. Carter, S.T. Blue. Data assimilation into nonlinear stochastic models. Tellus 51A, 167-194 (1999).

[22] J.T. Oden, T. Belytschko, J. Fish, T.J.R. Hughes, C. Johnson, D. Keyes, A. Laub, L. Petzold, D. Srolovitz, S. Yip. "Simulation-based engineering science: revolutionizing engineering science through simulation" in NSF Blue Ribbon Panel on SBES, 2006.

[23] F. Zhang, L. Xu, K. Zhang, E. Wang, J. Wang. The potential and flux landscape theory of evolution. The Journal of Chemical Physics, 137, 065102 (2012). 